\title{
Black hole holography and mean field evolution
}

\author{
David A. Lowe ${ }^{a}$ and Larus Thorlacius ${ }^{b, c}$ \\ ${ }^{a}$ Physics Department, Brown University, \\ Providence, RI, 02912, U.S.A. \\ ${ }^{b}$ University of Iceland, Science Institute, \\ Dunhaga 3, IS-10\%, Reykjavik, Iceland \\ ${ }^{c}$ The Oskar Klein Centre for Cosmoparticle Physics, Department of Physics, \\ Stockholm University, AlbaNova University Centre, \\ 10691 Stockholm, Sweden \\ E-mail: lowe@brown.edu, 1th@hi.is
}

ABSTRACT: Holographic theories representing black holes are expected to exhibit quantum chaos. We argue if the laws of quantum mechanics are expected to hold for observers inside such black holes, then such holographic theories must have a mean field approximation valid for typical black hole states, and for timescales approaching the scrambling time. Using simple spin models as examples, we examine the predictions of such an approach for observers inside black holes, and more speculatively inside cosmological horizons.

Keywords: AdS-CFT Correspondence, Black Holes, Models of Quantum Gravity

ArXIV EPRINT: 1710.03302 


\section{Contents}

1 Introduction 1

2 Scrambling in spin models $\quad 6$

$\begin{array}{lll}2.1 & \text { Modeling a young black hole } & 7\end{array}$

2.2 Modeling an old black hole 8

$\begin{array}{lll}2.3 \text { Summary } & 10\end{array}$

3 Mean field theory $\quad 10$

$\begin{array}{lll}3.1 & \text { Maximally entangled states in spin models } & 10\end{array}$

3.2 Saddle point analysis of the infinite-range Heisenberg model 11

$\begin{array}{ll}3.3 \text { Matching black hole parameters } & 13\end{array}$

\section{Introduction}

Holographic theories offer a way to define string theory nonperturbatively and address many of the outstanding questions in quantum gravity including the black hole information paradox. In a holographic setting the dual field theory provides a manifestly unitary description of gravitational dynamics that in principle extends to the formation and subsequent evaporation of black holes. The information about the microstate from which a black hole is formed must then be preserved throughout the evolution and released gradually to distant observers with the Hawking radiation emitted over the lifetime of the black hole.

In the present paper we address some key questions related to black holes in holographic theories, in particular the holographic representation of the black hole interior and near horizon region. Our main goal is to holographically model observations made in a laboratory entering a black hole in free fall and study deviations from bulk local quantum field theory caused by nonlocal holographic interactions. This follows up on and extends our previous work in $[1,2]$ on a toy model of black hole complementarity where local bulk evolution for infalling degrees of freedom emerges from a highly nonlocal holographic description via a mean field construction. The holographic degrees of freedom describing the black hole region are represented by a discrete collection of spin variables with long-range pairwise interactions whose strength is adjusted so as to obtain finite energy states in a large $N$ limit. With time the evolution of the mean field state at a given spin site will diverge from the evolution generated by the exact Hamiltonian of the spin system. This corresponds to the breakdown of the local semiclassical bulk description of an infalling observer inside a black hole due to holographic corrections and a central result of $[1,2]$ was that this breakdown happens on the same timescale as the observer encounters the curvature singularity. 
Our strategy is to focus on the degrees of freedom that describe the black hole interior and the near horizon region while eliminating those that describe the asymptotic region far away from the black hole. This is most economically represented by models with finite dimensional Hilbert spaces, which can always be expressed in terms of a finite number of interacting discrete spin variables. Our goal is to then extract predictions about how observables depend on the black hole microstate. The physics is governed by the interactions between the spins which are a priori unknown but should at the very least be such that the model exhibits fast scrambling [3] and the basic symmetries of the problem. In such models a version of global thermalization occurs on a timescale of order

$$
t_{s} \sim \frac{1}{k T} \log S,
$$

where $T$ is the Hawking temperature of the black hole and $S$ is the Bekenstein-Hawking entropy.

We note that our approach shares some common features with the Sachdev-Yi-Kitaev (SYK) model [4, 5], which has received considerable recent attention, but an important difference is that there one works in a low temperature limit where a conformal symmetry arises. That signals the model is describing an entire asymptotically anti-de Sitter spacetime, and one must then be careful to disentangle the asymptotic degrees of freedom from the black hole degrees of freedom in order to address the kind of questions we are interested in here. In the near horizon region and the interior of the black hole most of the symmetries of the asymptotic spacetime are broken. One has at best some remnant of rotational symmetry and an approximate time translation symmetry, assuming the black hole evaporates slowly, which constrains the possible interactions in the holographic Hamiltonian.

The unitary evolution of a black hole in asymptotically flat spacetime can be divided into four stages starting with its formation from matter in a pure state undergoing gravitational collapse. The classical geometry of the newly formed black hole will settle down fairly rapidly on a timescale of order the black hole mass in natural units into a quasistationary state, which for an uncharged and non-rotating black hole will be well described locally as a Schwarzschild geometry. This is followed by two stages of slow evaporation due to the emission of Hawking radiation. The two stages are distinguished by the nature of the quantum entanglement among the degrees of freedom that make up the black hole and outgoing Hawking radiation. During the earlier stage, often referred to as a young black hole, the majority of these degrees of freedom reside inside or near the remaining black hole and the entanglement entropy of the subsystem consisting of the Hawking radiation that has escaped from the near region is then very close to its maximal possible value. After the so-called Page time [6], when the area of the remaining black hole has been reduced to half its initial value, the roles are reversed and the Hawking radiation carries more than half the degrees of freedom. The state of the subsystem consisting of an old black hole, past its Page time, and its near environment is then maximally entangled with the long train of Hawking radiation that has already been emitted. The fourth stage comes at the end of the black hole lifetime when the Hawking temperature approaches the Planck temperature and no known gravitational theory can provide a reliable description of the physics. This 
is not a major concern however as presumably the violent final stage is very short lived and the black hole has already given up almost all its original quantum information before entering into it. In this work we will only be concerned with questions involving the two intermediate stages of slow evaporation but it will be important to distinguish between young and old black holes and their different entanglement structure in the holographic description of the interior geometry.

Hawking emission gradually transfers degrees of freedom from the black hole to outgoing radiation propagating in the far region and the black hole should strictly speaking be viewed as an open quantum system whose degrees of freedom are depleted with time. We can sidestep this complication since the characteristic timescale of the dynamics we are exploring is the scrambling time (1.1) and this is parametrically short compared to the black hole lifetime. Thus the number of Hawking quanta emitted in a scrambling time is very small compared to the total number of qubits required to describe the black hole. For our purposes here, it will thus be sufficient to consider a spin model with a fixed large number $N$ of spins, where $N$ is proportional to the Bekenstein-Hawking entropy of the remaining black hole. A young black hole can then be approximately represented by a pure state where the spins are maximally entangled, ignoring any error made by leaving out the degrees of freedom of the emitted Hawking radiation. In an old black hole on the other hand the spins encoding the black hole interior are entangled with the previously emitted Hawking radiation and are described by a maximally mixed spin state.

A class of spin models with highly nonlocal interactions was studied in [7] and it was shown that the resulting dynamics exhibits a form of fast scrambling for the states of interest. These models have $N$ discrete spins $\vec{\sigma}_{i}$ with pairwise interactions

$$
H=\sum H_{i j},
$$

where the sum ranges over unordered pairs of sites and the interactions are dense in the sense that the number of pairs with non-vanishing $H_{i j}$ scales as $N^{2}$. The interactions therefore have infinite range and the dynamics is insensitive to the dimension of the embedding spacetime. In order to have a sensible energy per degree of freedom in the large $N$ limit the strength of each two-body interaction must be bounded $\left\|H_{i j}\right\|<c / N$, where the norm $\|\mathcal{O}\|$ of an operator is given by the absolute value of its largest eigenvalue and $c>0$ is a constant.

Spin Hamiltonians of this type were further studied in [2] as simple holographic models of stretched horizon dynamics in the context of black hole complementarity. The local Hamiltonian dynamics in a radially infalling frame was modeled by a mean field Hamiltonian that depended on the initial state of the spin system. When the initial state is a product of two factors, one describing the black hole and the other representing a small "laboratory" entering the black hole in free fall, the decoherence between the local mean field evolution of the laboratory state and the exact holographic evolution takes place on timescales comparable to the scrambling time. This suggests that strong quantum gravity effects only occur well after the laboratory enters the black hole and not immediately upon crossing the horizon. The arguments presented in [2] apply to a class of spin models which is hopefully sufficiently generic to capture some of the behavior of actual holographic 
systems. The description is very crude however and to correctly describe the short-range Newtonian gravity limit of freely falling laboratories in detail would require a much more detailed specification of the holographic model, which for now we steer clear of.

In gauge/gravity duality in general, the map between the holographic model and the gravitational spacetime is extremely nonlocal. In the present case, we are already led to use a maximally nonlocal interaction between the spins to generate fast scrambling. We may view the spins as living on a lattice on a spherical surface embedded in the black hole spacetime. For example, we can consider approximately spherically symmetric states in four-dimensional spacetime, in which case the lattice will span the $\theta, \phi$ coordinates of a Schwarzschild solution.

The observables we will be interested in correspond to experiments conducted in freely falling laboratories, which we may then represent as a site on the lattice where the state is of the form

$$
|\psi\rangle=\left|\psi_{\text {lab }}\right\rangle \otimes\left|\psi_{\mathrm{bh}}\right\rangle
$$

where $\left|\psi_{\text {lab }}\right\rangle$ is a pure state of a spin on a lattice site. Here the term "spin" is used to represent any finite-dimensional Hilbert subspace, which encodes the full quantum state of the laboratory, and its onsite Hamiltonian can be arbitrary. For a young black hole $\left|\psi_{\mathrm{bh}}\right\rangle$ is a pure maximally entangled state on the remaining lattice sites. An old black hole on the other hand is maximally entangled with previously emitted Hawking radiation whose degrees of freedom are not included in the spin model and in this case the state $\left|\psi_{\mathrm{bh}}\right\rangle$ should be replaced by a density matrix describing a maximally mixed state.

Scrambling is accomplished via the nonlocal coupling between different sites. Time evolution with respect to the holographic Hamiltonian will evolve the state (1.3) forward in time. The main problem in mapping holographic time evolution to bulk time evolution involves correctly identifying the relevant bulk timeslices. In this regard, we follow [1] where a rather generic freely falling Planck lattice was considered as a bulk regulator. In [2] we found that many of the properties of holographic states are reproduced when matched with such a bulk description. On the bulk gravity side this evolution corresponds to propagating the laboratory inwards along a radial timelike geodesic. Since the holographic Hamiltonian eventually evolves the state toward a stationary thermal state, we are able to match the holographic time coordinate $t$ with the bulk Killing vector time, and we assume this matching can be carried out near the black hole a few Schwarzschild radii outside the horizon.

If we form the reduced density matrix of the laboratory site, by tracing over the Hilbert subspace associated with all other sites, the evolution will behave as an open quantum system $[8,9]$. In particular, the state $\left|\psi_{\text {lab }}\right\rangle$ will not experience local unitary evolution due to interactions with other sites. Ordinary local interactions with the surrounding spacetime account for part of this effect, but the dominant effect comes down to the nonlocal interaction with distant sites. One of the main questions we are interested in is to quantify the degree to which these nonlocal effects disrupt the experience of unitary quantum mechanics for the falling laboratory.

To study this we formulate a mean field approximation for the evolution of the state (1.3). Time dependent mean field approximations have previously been studied in 
interacting fermion systems in $[10,11]$. Such a mean field Hamiltonian by construction will be sitewise local, and Hermitian, and will be guaranteed to give a unitary evolution of the pure lab state $\left|\psi_{\text {lab }}\right\rangle$. However the choice of mean field Hamiltonian depends on the state $\left|\psi_{\text {bh }}\right\rangle$ so that the dynamics overall will not satisfy the superposition principle. By comparing the trace distance between the time evolution with respect to the exact Hamiltonian and the mean field Hamiltonian, one finds

$$
\left\|\rho_{\mathrm{lab}}(t)-\rho_{\mathrm{lab}}^{\mathrm{MF}}(t)\right\|_{1}<\frac{1}{S_{\mathrm{bh}}} e^{k T t}
$$

for a general class of spin models (matching $c=k T$ in the notation of [2]). We conclude that as long as $t<t_{s}$, with the scrambling time $t_{s}$ given by (1.1), then the nonlinear quantum mechanics violations of mean field are under control, and likewise the non-unitary effects of the exact evolution may be ignored. Thus for a finite time interval, the freely falling laboratory experiences the usual rules of quantum mechanics governed by the mean field Hamiltonian.

As we discuss more in the following, one may take a variety of different mean field Hamiltonians which are, in part, in correspondence with different choices of timeslicings of the bulk geometry. In previous work, we emphasized that there is a clear correspondence with slicings corresponding to freely falling coordinates (and close deformations thereof) where the timescale (1.1) arises as an upper bound on the time at which the laboratory hits the curvature singularity of the black hole geometry. Thus there seems to be a correspondence between singularity approach in the spacetime geometry and the breakdown of the mean field approximation in the holographic theory.

In the present paper our main goal is to study the rather detailed predictions that emerge for the corrections to local quantum field theory a freely falling laboratory will experience in the vicinity of a spacetime horizon. The clearest prediction is a shift of physical couplings in the laboratory by an amount that depends on the state of the black hole. We compute this in a number of examples where we find

$$
\delta H_{i i}=\operatorname{Tr}_{i^{c}} \rho_{\mathrm{MF}}(t) \sum_{j \neq i} H_{i j} \sim \frac{k T_{\mathrm{bh}}}{S_{\mathrm{bh}}^{1 / 2}} .
$$

Evaluating this for a four-dimensional Schwarzschild black hole the couplings shift by order $1 / M^{2}$ producing a timescale for observation of order $M^{2}$. This is significantly longer than the scrambling time, so will be difficult to detect by an interior observer [1]. On the other hand, this may lead to interesting effects for observers who remain outside the horizon, or observers in expanding universes who are potentially very long-lived.

Because the shift in the couplings depends on the microstate of the black hole, this may be viewed as a concrete realization of the soft hair proposal of $[12,13]$. To properly complete that idea, some regularization is needed to thin the infinite soft hair out to something that might account for the finite black hole entropy. The holographic construction in the present paper gives a nice example of such a regularization.

We also study the spectrum of maximally entangled states in the holographic theory. By construction, we are always free to add a constant to the Hamiltonian to match the 
overall energy with the bulk energy. More interesting is the distribution of states in the theory. With a suitable choice of model parameters we find the width of the energy spectrum to be $\sim k T$, corresponding to a highly degenerate black hole system with a spectral width of order the energy of a single Hawking particle.

We close with some thoughts on crossover models which will be a necessary generalization of these models if the goal is to generate both fast scrambling and the correct short-distance dynamics in the bulk. Broadly speaking one expects a spin-spin correlator to fall off on a length/timescale of order $1 / k T$ in a model with the correct short distance behavior. Such behavior can be generated by including both infinite range and nearest neighbor spin-spin interactions, and interesting critical points can be obtained by tuning the respective couplings. We hope to return to the consideration of such models in future work.

\section{$2 \quad$ Scrambling in spin models}

Quantum chaos in the usual sense is believed to appear in models with higher order interactions [5]. In the present work we will use a much simpler model based on pairwise interactions, and define our notion of scrambling using the trace distance between states, as in (1.4). Note that not all states will scramble rapidly in the models considered here. Moreover in the case of integrable spin models, there are an infinite number of conserved quantities, so many observables will not scramble at all. For the case at hand, we will be interested in a particular class of pure states that are maximally entangled, which exhibit fast scrambling even with simple integrable Hamiltonians. The main property we will need is simply the "infinite"-range interactions, coupling each site to every other.

In future, we hope to return to this set of questions in more complex models such as SYK, which in certain limits appear to saturate the scrambling time (at least when defined in terms of out-of-time-order four-point functions in a thermal state). The hope would be that in more general models generic initial states would scramble rapidly, in line with the conjectured behavior for the dual black holes. For the present, by restricting to initial states that are close to maximal entanglement, we can sidestep this issue, and still explore whether small laboratory subsystems still experience a breakdown of quantum mechanics with respect to their local mean field Hamiltonians.

In earlier work, Lieb-Robinson bounds have been used to derive bounds on the trace distance $[2,7,14]$. The advantage of this approach is that it works for any choice of pairwise Hamiltonian (with arbitrary "local" onsite couplings) and any choice of state. However one obtains only upper bounds on the behavior of the trace distance. Our first task in the present work is to see to what extent these bounds are saturated. We will find via direct numerical calculation that for sufficiently early times a bound of the form (1.4) is saturated. As the reduced density matrix approaches maximal mixing, the growth levels off, and exhibits Loschmidt echo phenomena [15-17]. In a more detailed model of quantum chaos, we expect this echo regime to be replaced by approximately constant trace distance indicative of a maximally mixed state, with recurrence happening over an inaccessible double exponential timescale $[18,19]$. However for the purposes of the present paper, 
where we focus on interior operators, it is sufficient to study the operators in the early time regime, only involving evolution approaching that of the scrambling time (1.1).

\subsection{Modeling a young black hole}

Having described the general setup, let us proceed to computation. In the following we will use the infinite-range Heisenberg model

$$
H=-\frac{J}{N} \sum_{i, j=1}^{N} \vec{s}_{i} \cdot \vec{s}_{j},
$$

where a spin $1 / 2$ degree of freedom on each site interacts with the rest. This is a particularly simple representative of the class of spin models in (1.2), with a uniform pairwise interaction and a single coupling constant $J$. Despite its simplicity the infinite-range Heisenberg model satisfies a basic requirement for scrambling that a local spin $\vec{s}_{i}$ does not commute with the Hamiltonian. For our initial state we take a state of the form (1.3) where $\left|\psi_{\text {lab }}\right\rangle$ is a pure state on a single lattice site that we label $i=0$ and $\left|\psi_{\mathrm{bh}}\right\rangle$ is chosen to be a maximally entangled state on the remaining $N$ sites. A maximally entangled state on an even number of sites may be constructed by taking a pairwise product of singlet states

$$
\left|\psi_{\max }\right\rangle=\bigotimes_{i=1}^{N / 2} \frac{1}{\sqrt{2}}\left(|\uparrow\rangle_{2 i-1}|\downarrow\rangle_{2 i}-|\downarrow\rangle_{2 i-1}|\uparrow\rangle_{2 i}\right) .
$$

Now one might be concerned this is a special choice of state. In general therefore we select a random unitary transformation $U_{\text {ran }}$ on the $2^{N}$ dimensional Hilbert space of the black hole sector, sampled with the natural unitary measure, and construct

$$
\left|\psi_{\mathrm{bh}}\right\rangle=U_{\mathrm{ran}}\left|\psi_{\max }\right\rangle
$$

The state $\left|\psi_{\mathrm{bh}}\right\rangle$ remains close to being maximally entangled, and may then be used to compute (1.4) for generic maximally entangled states.

In figure 1 the trace distance $N\left\|\rho_{\text {lab }}(t)-\rho_{\text {lab }}^{\mathrm{MF}}(t)\right\|_{1}$ is computed and averaged over random unitary matrices for values of $N \leq 14$. The reduced density matrix $\rho_{\text {lab }}(t)$ is obtained by evolving the system forward in time using the exact spin Hamiltonian (2.1). The mean field Hamiltonian that generates the evolution $\rho_{\mathrm{lab}}^{\mathrm{MF}}(t)$ is obtained in a selfconsistent fashion,

$$
\begin{aligned}
& H^{\mathrm{MF}}(t)=\sum_{i} H_{i}^{\mathrm{MF}}(t), \\
& H_{i}^{\mathrm{MF}}(t)=\operatorname{tr}_{\backslash \mathcal{H}_{i}}\left(H \rho^{\mathrm{MF}}(t)\right),
\end{aligned}
$$

where $\mathcal{H}_{i}$ is the Hilbert subspace associated with spin $i$, and $\backslash \mathcal{H}_{i}$ is the complement of this subspace. The mean field time evolution is then

$$
\partial_{t} \rho^{\mathrm{MF}}(t)=-i\left[H^{\mathrm{MF}}(t), \rho^{\mathrm{MF}}(t)\right]
$$




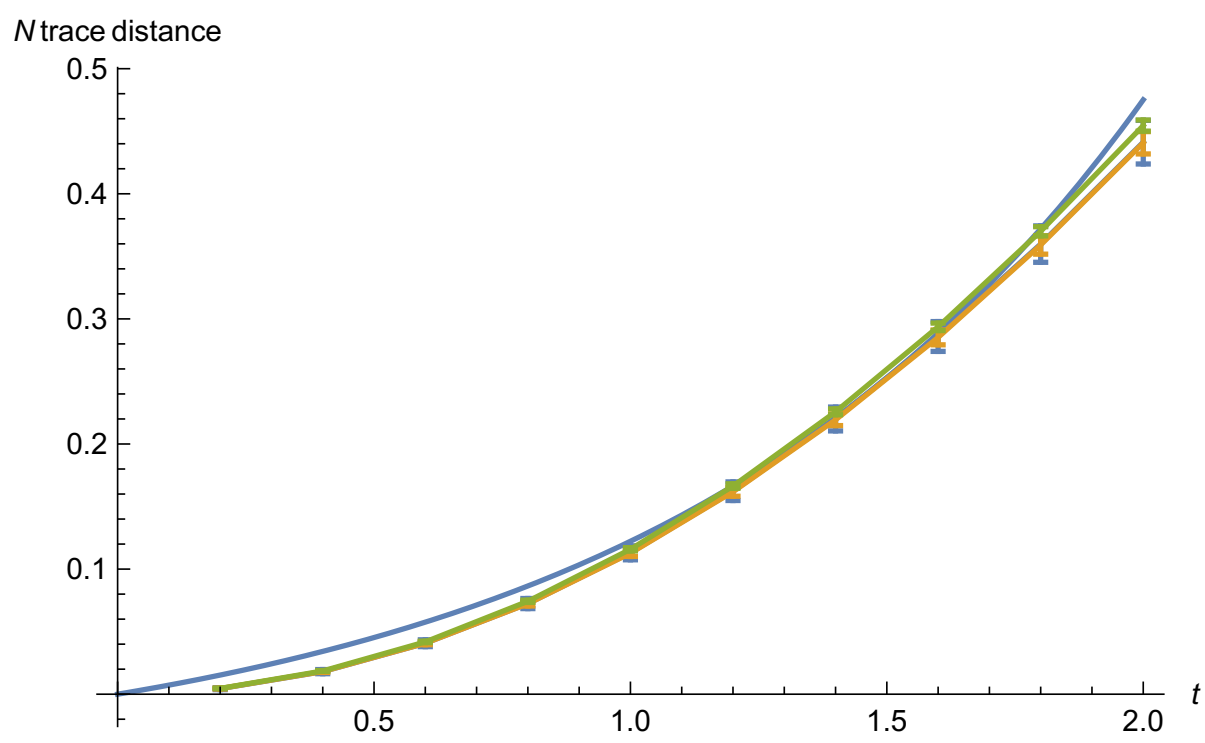

Figure 1. $N\left\|\rho_{\text {lab }}(t)-\rho_{\text {lab }}^{\mathrm{MF}}(t)\right\|_{1}$ is plotted for various values of $N \leq 14$. The error bars indicate an uncertainty due to picking different initial states. The numerical data is bounded by the curve $0.065(\exp (1.06 t)-1)$ shown in blue in the plot.

where $\rho^{\mathrm{MF}}(0)=\rho(0)$. The early time behavior shows a time dependence that converges as $N$ increases to an $N$-indepedent form. Likewise the error bars associated with the average over initial states are small. Finally the function is bounded by a function of the form $0.065(\exp (1.06 t)-1)$, allowing us to extract the scrambling time $t \sim \beta \log N$ as a characteristic timescale.

On somewhat longer timescales than shown in figure 1 the purity $\operatorname{Tr} \rho_{\text {lab }}^{2}(t)$, which starts at 1 , approaches that of a maximally mixed state (or $1 / 2$ for a 2 -state system). After this a Loschmidt echo develops, as shown in figure 2. If we identify the mixing timescale with the minimum of the purity, this is well fit by a logarithmic function $t_{p}=-1.70+4.26 \log N$ as shown in figure 3 , providing another way to see the scrambling time emerge from the evolution.

\subsection{Modeling an old black hole}

As a check on the previous results it is interesting to instead begin with the black hole sector in a maximally mixed density matrix. With the present computations, this is most easily accomplished by purifying the state. One way to accomplish this is to begin with the initial state (2.2) but set the Hamiltonian to vanish on sites indexed by $i$ even, and unchanged when $i$ is odd. The even sites then undergo trivial time evolution, but remain maximally entangled with the odd sites. Finally we may act with a random unitary transformation on the Hilbert subspace of the odd sites to generate a typical entangled state.

The results for $N_{\text {tot }} \leq 14$ (i.e. $N_{\text {bh }} \leq 7$ ) are shown in figure 4 . Up to numerical errors the results are the same as in the previous subsection for the early time behavior. Again this shows that $N\left\|\rho_{\text {lab }}(t)-\rho_{\text {lab }}^{\mathrm{MF}}(t)\right\|_{1}$ approaches an $N$ independent limit, that is bounded by a function of the form $0.061(\exp (1.04 t)-1)$. 


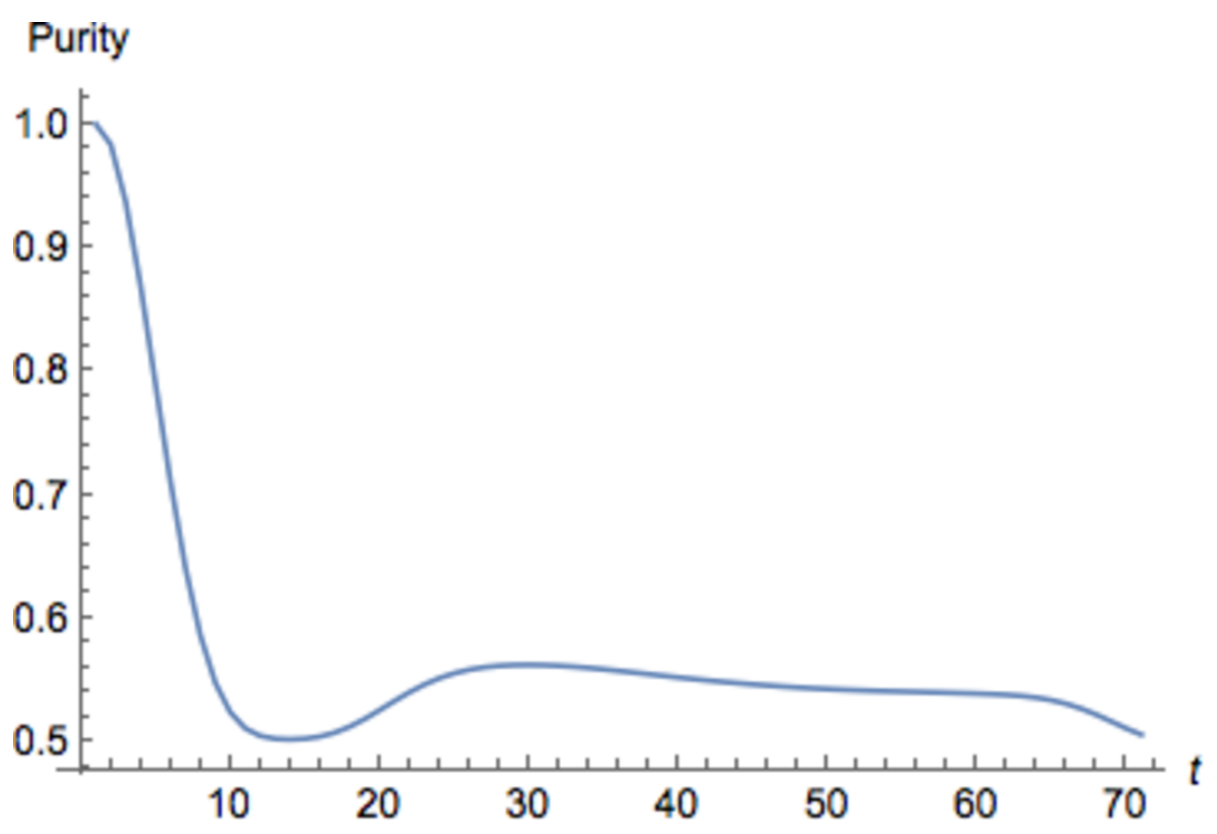

Figure 2. The purity $\operatorname{Tr} \rho_{\text {lab }}^{2}(t)$ decreases from 1 to close to maximal mixing. Then there is a partial recurrence, known as Loschmidt echo.

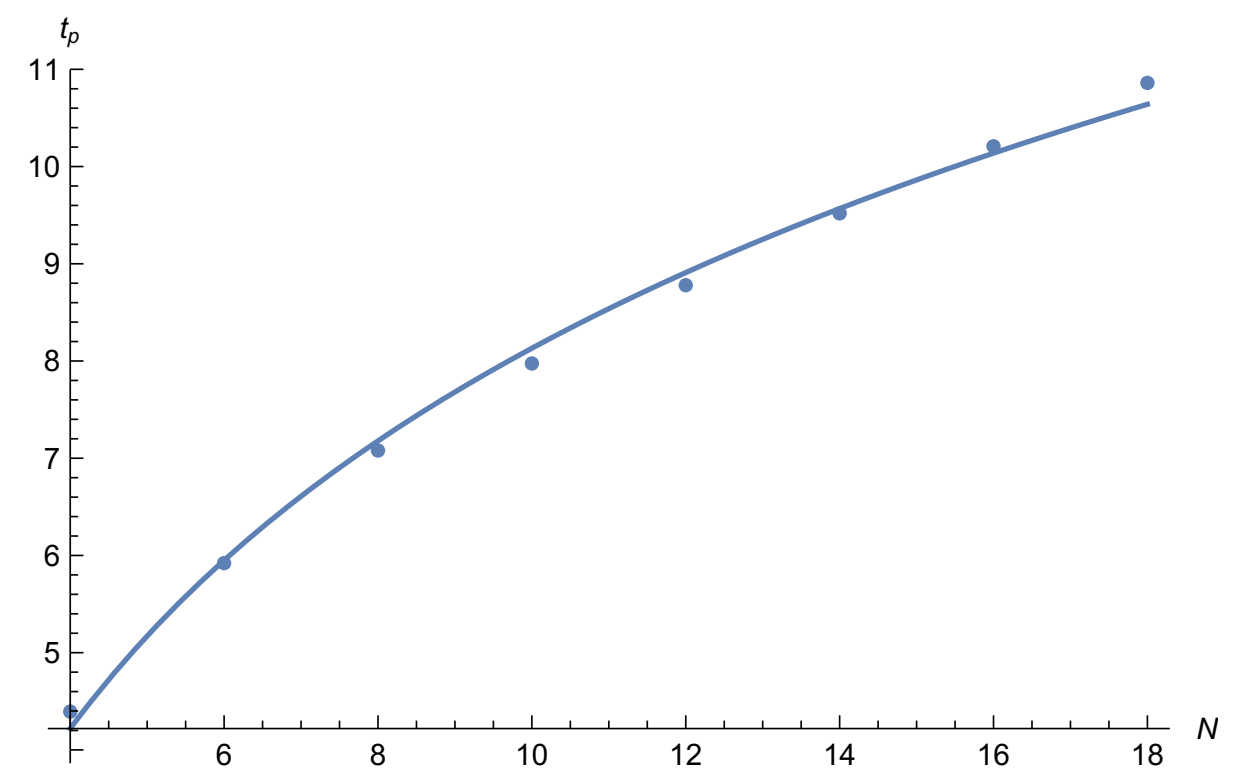

Figure 3. The mixing time defined by the minimum of the purity is plotted for different values of $N$. The blue line shows a fit to $t_{p}=-1.70+4.26 \log N$. 


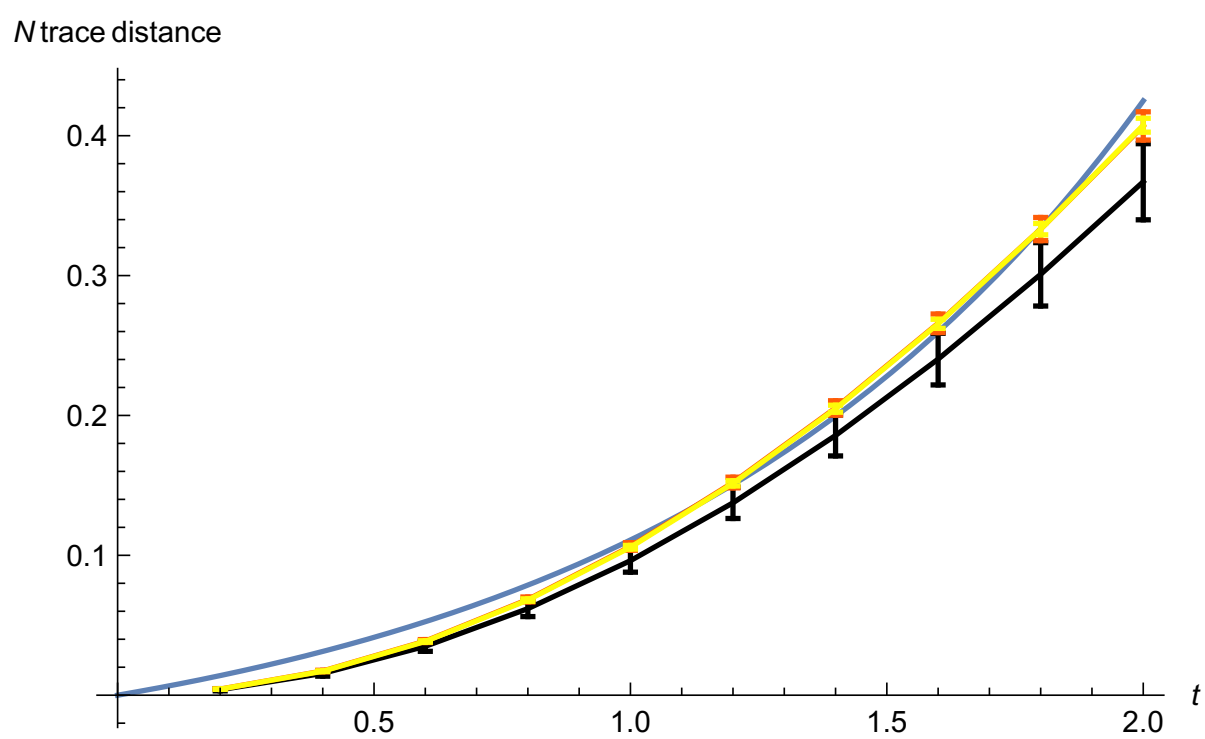

Figure 4. $\quad N\left\|\rho_{\text {lab }}(t)-\rho_{\text {lab }}^{\mathrm{MF}}(t)\right\|_{1}$ is plotted for maximally mixed black hole density matrices, representing an old black hole. The behavior is very similar to figure 1.

\subsection{Summary}

We have provided evidence that the early time behavior of the trace distance takes a universal form as $N$ becomes large. This comes close to saturating a bound of the form $\frac{a}{N}(\exp (b t)-1)$ where $a, b$ are $N$ independent constants. The scrambling time $t \sim \log N$ then naturally emerges from this construction.

\section{Mean field theory}

We now wish to study observables in the class of states described in the previous section. One approach would be to carry out explicit numerical calculations but, as we have seen above, the rapid growth of Hilbert space dimensions and limited computer power restricts this to relatively modest values of $N$. We instead adopt a strategy that allows explicit analytic calculations that are valid for large $N$. The first step is to reformulate the states of interest as unentangled pure states in a coarse grained spin model and then carry out a standard thermodynamic analysis.

\subsection{Maximally entangled states in spin models}

A maximally entangled state is unitary equivalent to a product state of the form (2.2), where for simplicity we imagine each site contains a spin $1 / 2$ degree of freedom. As shown in [2], we may start with a generic maximally entangled state and perform a unitary transformation to get such a state. Such a unitary transformation does not necessarily commute with the Hamiltonian.

Now if we coarse grain, combining the sites $k$ and $k+1$ into a single site, and the state on each pair into a pure state on the coarse-grained lattice, we are left with an unentangled 
pure state

$$
|\psi\rangle=\prod_{k=2 i}|\chi\rangle_{k}
$$

For the purposes of calculating generic observables such as the energy spectrum it should be sufficient to consider the coarse grained spin model in a generic unentangled state. As will become clear below, it is in fact sufficient to consider the spin model in a high temperature limit to compute the observables of interest. We focus our attention on the infinite-range Heisenberg model as a representative of the more general class of nonlocal spin models (1.2). In the previous section we presented numerical evidence that even in this simple model the nonlocal spin dynamics fast scrambles maximally entangled states. In the following we carry out a thermodynamic analysis of the energy spectrum and argue that with the appropriate choice of parameters it matches the spectrum of an evaporating black hole.

\subsection{Saddle point analysis of the infinite-range Heisenberg model}

Consider $N$ spins interacting via the Hamiltonian (2.1) where the spin operator $\overrightarrow{s_{i}}$ generates an $\mathrm{SU}(2)$ rotation on a two-component qubit at site $i$ and $J>0$ is a constant that will be adjusted later on to match the behavior of black hole states. For convenience we have included diagonal terms with $i=j$ in the double sum in the Hamiltonian. These terms contribute a constant to the total energy and by including them the Hamiltonian is simply the square of the total spin,

$$
H=-\frac{J}{N}\left(\sum_{i=1}^{N} \vec{s}_{i}\right)^{2}
$$

The energy eigenvalues are given by $E_{S}=-J S(S+1) / N$ where the total spin quantum number $S$ is integer spaced and ranges from $N / 2$ down to 0 or $1 / 2$ depending on whether $N$ is even or odd.

The finite temperature behavior of the system is encoded in the partition sum,

$$
Z[\beta]=\operatorname{Tr}\left[e^{-\beta H}\right]=\sum_{S} d(S, N) e^{\beta J S(S+1) / N},
$$

where $d(S, N)$ is the number of states with total spin $S$ appearing in the $N$-fold tensor product of fundamental representations [20],

$$
d(S, N)=\frac{(2 S+1)^{2}}{N+1}\left(\begin{array}{c}
N+1 \\
\frac{N}{2}-S
\end{array}\right)
$$

At a fixed large value of $N$ the number of states has a maximum at $S \simeq \sqrt{N / 2}$ and the partition sum is dominated by terms where both arguments of the binomial coefficient are large. In this case we can use Stirling's approximation,

$$
\left(\begin{array}{c}
N+1 \\
\frac{N}{2}(1-x)
\end{array}\right) \simeq \frac{2^{N+\frac{3}{2}}}{\sqrt{\pi N}}(1-x)^{-1 / 2}(1+x)^{-3 / 2} e^{-\frac{N}{2}[(1-x) \log (1-x)+(1+x) \log (1+x)]},
$$




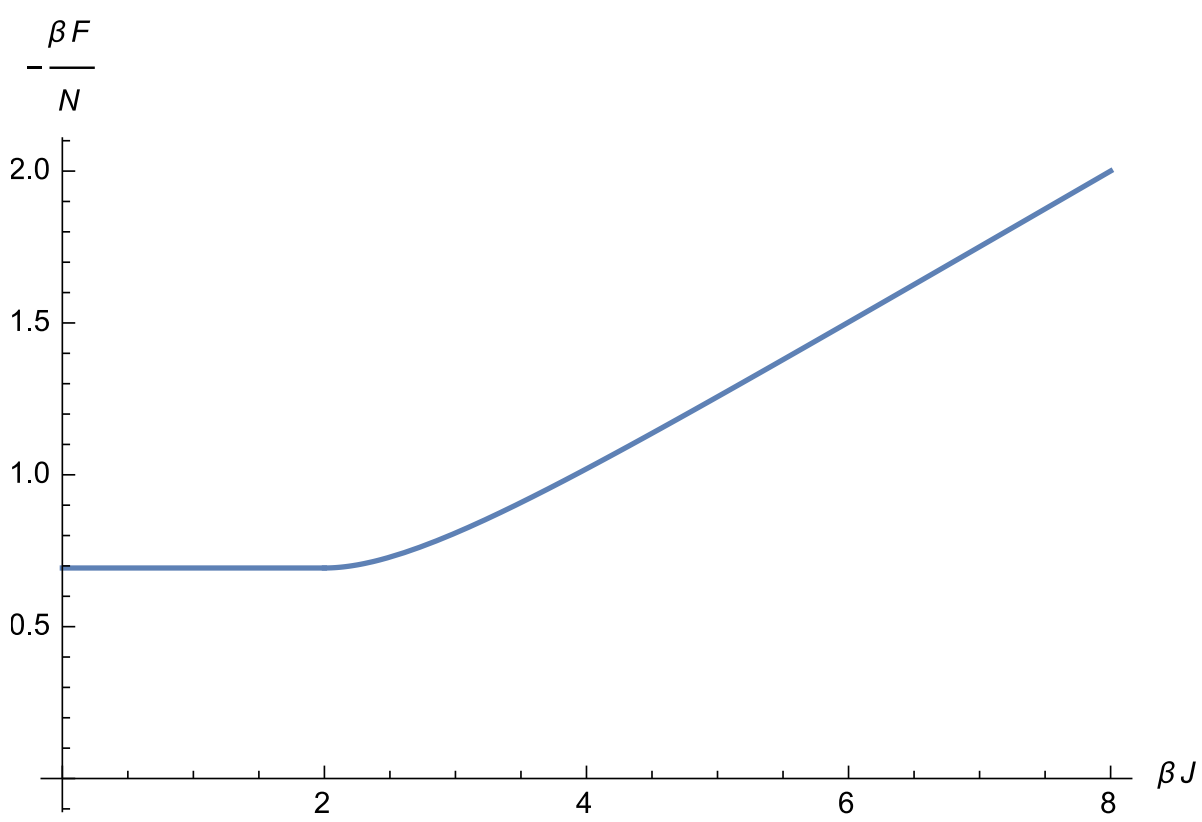

Figure 5. Free energy per site at large $N$ in the infinite range Heisenberg model.

where $x=2 S / N$. The sum over $S$ can then be approximated by an integral over $x$,

$$
Z[\beta]=\frac{2^{N+\frac{1}{2}} N^{\frac{3}{2}}}{\sqrt{\pi}} \int_{0}^{1} d x f(x) e^{N g(x)},
$$

with

$$
f(x)=\frac{\left(x+\frac{1}{N}\right)^{2} e^{\beta J x / 2}}{(1-x)^{1 / 2}(1+x)^{3 / 2}},
$$

and

$$
g(x)=\frac{1}{4} \beta J x^{2}-\frac{1}{2}(1-x) \log (1-x)-\frac{1}{2}(1+x) \log (1+x) .
$$

The integral can be evaluated in a saddle point approximation valid at large $N$. The resulting saddle point equation,

$$
x=\tanh \left(\frac{1}{2} \beta J x\right),
$$

reveals a second order phase transition at a critical temperature $k T_{c}=J / 2$.

In the low temperature phase $\beta J>2$ the saddle point equation has a non-trivial solution at $x=x_{*}>0$ and the free energy is given by

$$
-\beta F=\log Z=N\left(\log 2+g\left(x_{*}\right)\right)+O(\log N) .
$$

Figure 5 plots the saddle point value of the free energy per site, as a function of the dimensionless variable $\beta J$ in the limit of large $N$.

It is, however, the high temperature limit $\beta J \ll 1$ that is of interest for modeling black hole behavior. In the high temperature phase the saddle point sits at the endpoint of the 
integration range $x=0$ and in this case the leading large $N$ contribution to the free energy is simply

$$
-\beta F=\log Z=N \log 2+\cdots .
$$

The energy vanishes at leading order,

$$
U=-\frac{\partial}{\partial \beta} \log Z=0+\cdots,
$$

and the entropy is given by

$$
S=k\left(1-\beta \frac{\partial}{\partial \beta}\right) \log Z=k N \log 2+\cdots .
$$

We need to include subleading $N^{0}$ terms in our saddle point analysis in order to see how the thermodynamic variables depend on temperature. A straightforward calculation gives

$$
\begin{aligned}
-\beta F & =N \log 2-\frac{3}{2} \log \left(1-\frac{\beta J}{2}\right), \\
S & =k N \log 2-\frac{3 k}{2} \log \left(1-\frac{\beta J}{2}\right)-\frac{3 k \beta J}{4-2 \beta J}, \\
U & =-\frac{3 J}{4-2 \beta J} .
\end{aligned}
$$

The negative sign energy is consistent with $H$ in equation (2.1) being negative definite. We note that the entropy scales linearly with the number of sites in the high temperature limit $\beta J \rightarrow 0$ while the width of the energy spectrum remains finite at large $N$,

$$
(\Delta U)^{2}=\frac{\partial^{2}}{\partial \beta^{2}} \log Z=\frac{3 J^{2}}{2(2-\beta J)^{2}} .
$$

\subsection{Matching black hole parameters}

If we shift $U$ by a constant so as to match the black hole energy $(U=M)$ and dial the coupling so that

$$
J \sim k T_{\mathrm{bh}}
$$

corresponding to matching the scrambling time in the model [2] to (1.1), then the high temperature phase of this simple spin model exhibits precisely the sort of highly degenerate energy spectrum that one expects for an evaporating black hole. In particular the width of the black hole states, which is independent of the constant shift in $U$, matches that of a Hawking quantum.

The self consistency of considering the high temperature phase $(\beta J \ll 1)$ of the spin model can be checked as follows. The entropy scales linearly with the number of sites and is also proportional to the black hole area, $N \sim S \sim r_{s}^{D-2}$, where $r_{s}$ is the Schwarzschild radius and $\mathrm{D}$ is the spacetime dimension. The black hole temperature scales as $T_{\mathrm{bh}} \sim r_{s}^{-1} \sim$ $N^{-1 /(D-2)}$ and thus the identification (3.1) ensures that $\beta J \rightarrow 0$ in the large $N$ limit for any finite value of $\beta$. In other words the spin model is effectively in a high temperature limit for the parameter values that match the black hole physics we wish to model. 
The above results may also be used to infer the mean field shift in the onsite Hamiltonian,

$$
\left(\Delta H_{i i}\right)^{2}=\frac{1}{N}(\Delta U)^{2} \sim \frac{\left(k T_{\mathrm{bh}}\right)^{2}}{S_{\mathrm{bh}}} .
$$

The onsite Hamiltonian is to be viewed as the worldline Hamiltonian for a free falling observer in the bulk spacetime. Therefore this shift (3.2) gives an estimate of the size of the potentially observable effect a local observer can detect that arises from the nonlocal holographic physics of the exact evolution. For black holes, this kind of energy shift will be hard to measure in free fall since the lifetime of such an infalling observer with respect to the holographic time will be bounded by (1.1) by the arguments of [1]. However an exterior observer who is long-lived, and able to conduct experiments on the stretched horizon should be able to avoid such a restriction. In this case, such an observer can potentially distinguish the microstates of the black hole. One can perhaps view this dependence of local observables on the black hole microstates as a concrete realization of the soft hair proposal of [12].

It is also interesting to apply the above kind of reasoning to a de Sitter horizon. Let us for the sake of argument assume that a similar holographic description involving a finite dimensional spin model can be developed for the degrees of freedom associated with a stretched de Sitter horizon. In this case a freely falling observer inside a Hubble volume can in principle live long enough to make the observation of energy shifts analogous to (3.2) observable, allowing a de Sitter observer to gain information about the horizon microstate. The analogy with the black hole problem leads to a more worrying concern that local quantum mechanics will break down for such an observer after a de Sitter scrambling time $t_{s} \sim L_{d S} \log \left(L_{d S}\right)$. The construction of a convincing holographic description of de Sitter spacetime remains an open problem. We hope to return to this issue in future work.

\section{Acknowledgments}

This research was supported in part by DOE grant de-sc0010010, Icelandic Research Fund grant 163422-052, the University of Iceland Research Fund, and the Swedish Research Council under contract 621-2014-5838. The authors also wish to thank the Simons Center for Geometry and Physics, Stony Brook University, for hospitality and support during the final stages of the project.

Open Access. This article is distributed under the terms of the Creative Commons Attribution License (CC-BY 4.0), which permits any use, distribution and reproduction in any medium, provided the original author(s) and source are credited.

\section{References}

[1] D.A. Lowe and L. Thorlacius, Quantum information erasure inside black holes, JHEP 12 (2015) 096 [arXiv: 1508.06572] [INSPIRE].

[2] D.A. Lowe and L. Thorlacius, A holographic model for black hole complementarity, JHEP 12 (2016) 024 [arXiv: 1605.02061] [INSPIRE]. 
[3] Y. Sekino and L. Susskind, Fast Scramblers, JHEP 10 (2008) 065 [arXiv:0808.2096] [INSPIRE].

[4] S. Sachdev and J. Ye, Gapless spin fluid ground state in a random, quantum Heisenberg magnet, Phys. Rev. Lett. 70 (1993) 3339 [cond-mat/9212030] [INSPIRE].

[5] A. Kitaev, A simple model of quantum holography, KITP strings seminar and Entanglement $2015(2015)$.

[6] D.N. Page, Average entropy of a subsystem, Phys. Rev. Lett. 71 (1993) 1291 [gr-qc/9305007] [INSPIRE].

[7] N. Lashkari, D. Stanford, M. Hastings, T. Osborne and P. Hayden, Towards the Fast Scrambling Conjecture, JHEP 04 (2013) 022 [arXiv:1111.6580] [INSPIRE].

[8] E.B. Davies, Quantum Theory of Open Systems, Academic Press Inc. (1976).

[9] F. Benatti, Dynamics, Information and Complexity in Quantum Systems, first edition, Springer Publishing Company (2009).

[10] C. Bardos, F. Golse, A.D. Gottlieb and N.J. Mauser, Mean field dynamics of fermions and the time-dependent hartree-fock equation, J. Math. Pure. Appl. 82 (2003) 665.

[11] N. Benedikter, M. Porta and B. Schlein, Mean-field evolution of fermionic systems, Commun. Math. Phys. 331 (2014) 1087.

[12] S.W. Hawking, M.J. Perry and A. Strominger, Soft Hair on Black Holes, Phys. Rev. Lett. 116 (2016) 231301 [arXiv:1601.00921] [INSPIRE].

[13] S.W. Hawking, M.J. Perry and A. Strominger, Superrotation Charge and Supertranslation Hair on Black Holes, JHEP 05 (2017) 161 [arXiv: 1611.09175] [INSPIRE].

[14] D.A. Lowe and L. Thorlacius, Black hole complementarity: The inside view, Phys. Lett. B 737 (2014) 320 [arXiv: 1402.4545] [INSPIRE].

[15] A. Peres, Stability of Quantum Motion in Chaotic and Regular Systems, Phys. Rev. A 30 (1984) 1610 [INSPIRE].

[16] R.A. Jalabert and H.M. Pastawski, Environment-independent decoherence rate in classically chaotic systems, Phys. Rev. Lett. 86 (2001) 2490.

[17] B. Žunkovič, A. Silva and M. Fabrizio, Dynamical phase transitions and loschmidt echo in the infinite-range xy model, Philos. Trans. Roy. Soc. London A 374 (2016) 2069.

[18] P. Bocchieri and A. Loinger, Quantum recurrence theorem, Phys. Rev. 107 (1957) 337.

[19] D.N. Page, Information loss in black holes and/or conscious beings?, hep-th/9411193 [INSPIRE].

[20] A.W. Aste, The finite infinite range Heisenberg model and microcanonical black hole statistics, arXiv:1502.00844 [INSPIRE]. 